\title{
Objective crystallographic symmetry classifications of two membrane proteins
}

\author{
Peter Moeck
}

Portland State University, Portland, Oregon, United States

Methods for objective classifications of more or less 2D periodic patterns into Bravais lattice types, Laue classes, and plane symmetry groups have recently been developed [1,2]. The objectivity of the crystallographic symmetry classifications is ensured by the statistically sound selection of the best geometric model for the 2D periodic signal in the image data on the basis of geometric Akaike Information Criteria [3]. As recently reviewed [4], the new crystallographic image classification methods are the only ones that can be considered to be objective, i.e. researcher and arbitrary thresholds independent. These techniques are analytic in nature rather than based on machine learning. This feature enables them to deal effectively with all types of pseudosymmetries and to obtain geometric Akaike weights, which represent the probabilities of the correctness of particular crystallographic symmetry classifications. (Machine learning systems have so far ignored pseudosymmetries in crystallographic image classification studies.)The digital input images of the more or less 2D periodic patterns are considered to consist of the pixel-wise sums of more or less Gaussian distributed noise and an unknown underlying signal that is strictly 2D periodic. Structural defects in the molecular 2D array, instrumental image recording noise, small systematic errors, and small inaccuracies in the algorithmic processing of the image data all contribute to a single generalized noise term. Because there are many different sources of noise and only small systematic errors that all contribute to the generalized noise term, the central limit theorem justifies the overarching assumption that the generalized noise is approximately Gaussian distributed.Experimental images from transmission electron microscopes that are digital and sufficiently well resolved at the molecular level serve as the input of the crystallographic symmetry classifications in electroncrystallography based structural biology.For good information extraction results and generalized noise suppression, one should record experimental images with a large field of view, containing several hundreds of more or less identical unit cells of the 2D periodic array. The outputs of the techniques are the most probable 2D periodic signal distribution from the underlying molecular array in addition to the most probably plane symmetry group, Laue class, and Bravais lattice type. The generalized noise level is quantified as a byproduct. The new methods are demonstrated on (nominal) zero-tilt transmission electron microscope images from tilt series of 2D crystals of a fragment of beef heart NADH:ubiquinone oxidoreductase and a prokaryotic cyclic nucleotide-modulated potassium channel.

\section{References}

[1] P. Moeck, Symmetry, vol. 10, paper 133 (46 pages), 2018, doi: 10.3390/sym10050133, open access.

[2] A. Dempsey and P. Moeck, arXiv:2009.08539, December 15, 2020, open access.

[3] K. Kanatani, IEEE Trans. Pattern Analysis Machine Intelligence 26, 1307-1319 (2004), doi: 10.1109/TPAMI.2004.93.

[4] P. Moeck, IEEE Trans. Nanotech. 18, 1166-1173 (2019), doi: 10.1109/TNANO.2019.2946597. 